\title{
ADV score is a quantifiable prognostic prediction model for surgical resection of hepatocellular carcinoma: A Korea-Japan collaborative validation study with 10,606 patients
}

\author{
Shin Hwang*
}

Department of Surgery, Asan Medical Center, University of Ulsan College of Medicine, Seoul, Korea

Background: We previously demonstrated that multiplication of $\alpha$-fetoprotein (AFP), des- $\gamma$-carboxy prothrombin (DCP) and tumor volume (TV) (ADV score, expressed in log10) is an integrated surrogate marker of post-resection prognosis for hepatocellular carcinoma (HCC). This study aimed to validate the ADV score as a quantifiable biomarker for oncological aggressiveness of HCC on its prognostic impact following resection of HCC.

Methods: A multicenter validation cohort with 10,606 patients (4,900 [46.2\%] from Korea and 5,706 [53.8\%] from Japan) was established through the Korea-Japan collaboration study groups managed by the Korea and Japan Society of Hepatobiliary Pancreatic Surgery.

Results: In the Korean cohort, disease-free survival (DFS) and overall survival (OS) rate at 5 years were $66.7 \%$ and $93.0 \%$ in ADV score < 2log; $56.0 \%$ and $84.1 \%$ in ADV score 2.0-2.9log; 51.0\% and 79.7\% in ADV score 3.0-3.9log; 45.7\% and 75.3\% in ADV score 4.04.9log; 44.4\% and 69.4\% in ADV score 5.0-5.9log; 38.4\% and 64.3\% in ADV score 6.0-6.9log; $32.6 \%$ and 62.9\% in ADV score 7.0-7.9log; $28.7 \%$ and $53.2 \%$ in ADV score $8.0-8.9 \log$; $30.4 \%$ and $46.5 \%$ in ADV score $9.0-9.9 \log$; $30.5 \%$ and $47.7 \%$ in ADV score $10.0-10.910$; 22.5\% and 35.7\% in ADV score 11.0-11.9log; and 18.1\% and 33.8\% in ADV score $\geq 12 \mathrm{log}$, respectively (both $p<0.001$ ). The ADVscore dependent DFS and OS rates in the Japanese cohort were very similar with those of the Korean cohort. Consequently, the overall study cohort also revealed similar ADV-score dependent DFS and OS rates. Multiplication of AFP and DCP (AD score) and TV were independent risk factors for DSF and OS. The 5-year DFS and OS rates were visualized according to AD score and TV as like weather prediction diagrams.

Conclusions: This high-volume international validation study revealed that the preoperative ADV score is a reliable surrogate biomarker for quantifiable prediction of post-resection prognosis in the Korean and Japanese patients with HCC. 